\title{
Morphological Measurements of Anatomic Landmarks in Human Mandibular Molar Pulp Chambers - An in vivoStudy
}

\author{
Joyti Lokade', Shivlal Rawlani², Rakhi Baheti (Chandak)², Shelly Roy', \\ Manoj Chandak', Vidya Lohe ${ }^{2}$
}

1. Department of Conservative Dentistry, Sharad Pawar Dental College, DMIMS, Sawangi (M), Wardha, India

2. Department of Oral Medicine \& Radiology, Sharad Pawar Dental College, DMIMS, Sawangi (M), Wardha, India

\section{Corresponding Author}

\author{
Shivlal Rawlani, MDS \\ Department of Oral Medicine \& Radiology, Sharad Pawar Dental College, DMIMS, Sawangi (M), \\ Wardha, Maharashtra, India \\ E-mail : drrawlani2007@rediffmail.com
}

Received for publication Jan 12, 2011; Returned after revision Mar 13, 2011;

Accepted for publication Apr 14, 2011

\begin{abstract}
- Abstract
Purpose: Exact knowledge of the location and dimension of the pulp chamber help to maintain the pulp healthy during operative procedure and also reduces the risk of perforation of pulp chamber during root canal treatment. This in-vivo study was carried out to measure critical morphology of pulp chamber of mandibular molar using intra-oral periapical radiograph.

Materials and Methods: Mandibular molar teeth of 56 patients were evaluated. The mandibular molar teeth whose pulp chamber was not violated by caries, restoration, fracture crown and those having closed apex were included in the study. The intraoral periapical radiographs were taken with paralleling angle technique using radio-opaque grid with $1 \mathrm{~mm}$ space. This grid was placed directly on the film.

Results: In $94 \%$ of the mandibular first molars specimens the pulp chamber ceiling was at the level of the cemento-enamel junction. The measurements showing the lowest percentage variance were buccal cusp to furcation (approximately 11\%) and buccal cusp to pulp chamber ceiling (approximately 15\%). The distance from the cusp tip to pulp chamber ceiling height was approximately $6.0 \mathrm{~mm}$, the distance from the pulpal floor to the furcation was approximately $3.0 \mathrm{~mm}$, and the average height of a pulp chamber was 1.5 to $2.0 \mathrm{~mm}$.

Conclusion: The exact knowledge of distances of pulp chamber from various anatomical landmarks helps in proper assessment of root canals and ultimately avoids the failure of root canal treatment.
\end{abstract}

- Key word : Mandibular first molar, Dental pulp cavity, Cementoenamel junction, Furcation defects

- J Kor Dent Sci. 2011; 4(1): 1 - 5

(c) This is an open access article distributed under the terms of the Creative Commons Attribution Non-Commercial License (http:// creativecommons.org/licenses/by-nc/3.0) which permits unrestricted non-commercial use, distribution, and reproduction in any medium, provided the original work is properly cited. 


\section{Introduction}

The preservation of pulp in healthy state during operative procedure and successful management of pulp in case of diseases are two most important challenges in day today practice for clinician ${ }^{1)}$. Commonly occurring complications during endodontic treatment is perforation into the furcation area during access preparation of molar teeth which often results in failure of treatment ${ }^{2)}$.

Perforation through the floor of the pulp chamber into the furcation of any tooth is a serious problem. The outcome of perforation repair is not predictable and most of the times result in extraction of the tooth ${ }^{2)}$. Access preparations are performed by a qualitative method involving the clinician's tactile perception and knowledge of dental anatomy. However, a reliance on tactile perception alone may lead to undesirable results ${ }^{3)}$. Calcification of the pulp cavity may reduce tactile perceptions, leading to iatrogenic failure ${ }^{4,5)}$. In this situation, the pulp chamber roof and floor approximate each other, causing complication.

A review of the literature regarding the morphology of the pulp chamber revealed very less information. Conversely, few studies describe the morphology of the furcation as it relates to the floor of the pulp chamber. A study conducted by Sterrett and Pelletier ${ }^{6}$ measured the distance from the floor of the pulp chamber to five predetermined sites on the furcation root surface and found it to range from 2.7 to 3.0 $\mathrm{mm}$ for both mandibular and maxillary molars .

While the study conducted by Majzoub ${ }^{7}$ reported that the mean distance from the pulp chamber floor to the root separation of maxillary molars was equal to or less than 3 $\mathrm{mm}$ in $86 \%$ of the teeth measured. Clearly, knowledge of

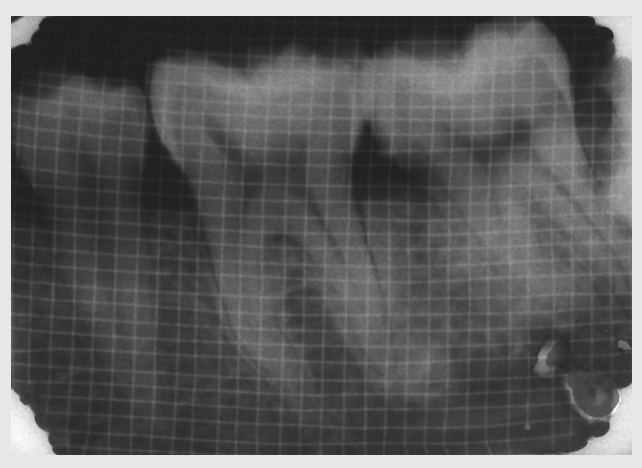

Figure 1. Radiograph showing superimposition of grid.

Joyti Lokade, et al: Morphological Measurements of Anatomic Landmarks in Human Mandibular Molar Pulp Chambers - An in vivo Study. J Kor Dent Sci 2011. variations in dimensions of the molar pulp chamber may reduce perforations of the chamber during treatment.

Instead of completely relying on tactile sense, external anatomical landmark can be taken into consideration. These landmarks can act as predictor for location of roof and floor of pulp chamber and their interrelations. Hence this in-vivo study was carried out with an aim to measure external anatomical landmark and their co-relation with critical pulp chamber morphology in mandibular molar using intraoral periapical radiograph.

\section{Materials and Methods}

The study was carried out in the department of conservative dentistry and department of oral medicine and radiology. After getting approval from ethical board, all the patients included in the study were explained about the complete study and a written consent was obtained.

Fifty-six patients in the age range of 20 25 yrs (Mean age 23 years) with mandibular molar were selected for this study. Only those teeth whose pulp chamber was not violated by caries, restoration, fracture crown and having closed apex were considered in to present study.

The intraoral periapical radiographs were taken using paralleling angle technique with GNATUS Raios X Timex intraoral machine (70 kv, 7 MA, Gnatus, Ribeiräo Preto, Brazil). The radio-opaque grid with $1 \mathrm{~mm}$ space was placed directly on the film. When the radiograph was exposed, the $1 \mathrm{~mm}$

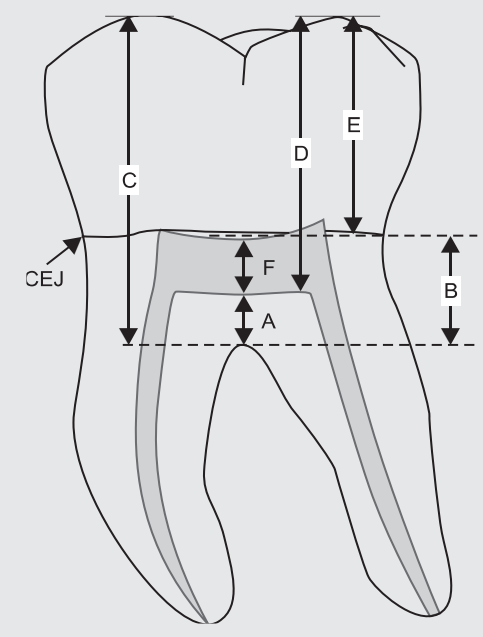

Figure 2. Showing distance of pulp chamber from various anatomical landmarks.

Joyti Lokade, et al: Morphological Measurements of Anatomic Landmarks in Human Mandibular Molar Pulp Chambers - An in vivo Study. J Kor Dent Sci 2011. 
grid gets superimposed on the radiographic image of the anatomic area of interest providing a reference for measurements.

Measurements were taken by a single operator. Three direct measurements were taken for each tooth (Fig. 1, 2).

Measurement- A: Represents the distance between the floor of the pulp chamber and the closest point to the furcation.

Measurement- B: Represents the distance from the ceiling of the pulp chamber to the closest point to the furcation.

Measurement- C: Represents the distance from the buccal cusp tip to the closest point to the furcation.

Measurement- D: Represents the distance from the buccal cusp to the pulp chamber floor.

Measurement- E: Represents the distance from the buccal cusp to the pulp chamber ceiling.

Measurement- F: Represents the height of pulp chamber. The location of the pulp chamber ceiling with respect to the level of cementoenamel junction was also evaluated and recorded. All the measurements were recorded and by using descriptive statical analysis were done. Mean and standard deviation was obtained for each measurement.

\section{Results}

The mean, SD, and coefficient of variation for each measurement are presented for mandibular first molars (Table 1). It should be noted that means for all the measurements are very similar. In the present study $94 \%$ of the cases show pulp chamber ceiling at the level of the cementoenamel junction in mandibular molars.

Table 1. Various measurements in relation to pulp chamber

\begin{tabular}{|c|c|c|c|c|c|c|}
\hline & A & B & C & $D$ & $E$ & $\mathrm{~F}$ \\
\hline Mean (mm) & $2.32 \pm 0.47$ & $3.80 \pm 0.77$ & $9.08 \pm 1.03$ & $6.83 \pm 0.96$ & $5.37 \pm 0.79$ & $1.48 \pm 0.66$ \\
\hline$\% \mathrm{CV}$ & 20.3125 & 20.33 & 11.35 & 14.155 & 15.07 & 44.61 \\
\hline \multicolumn{7}{|c|}{$\begin{array}{l}\text { A: distance between the floor of the pulp chamber and the closest point to the } \\
\text { furcation, B: distance from the ceiling of the pulp chamber to the closest point to } \\
\text { the furcation, C: distance from the buccal cusp tip to the closest point to the } \\
\text { furcation, D: distance from the buccal cusp to the pulp chamber floor, E: distance } \\
\text { from the buccal cusp to the pulp chamber ceiling. F: Represents the height of pulp } \\
\text { chamber. } \\
\text { \% CV. Coefficient of Variance. }\end{array}$} \\
\hline
\end{tabular}

Joyti Lokade, et al: Morphological Measurements of Anatomic Landmarks in Human Mandibular Molar Pulp Chambers - An in vivo Study. J Kor Dent Sci 2011.

\section{Discussion}

A review of the endodontic literature contains relatively few studies that actually measure the anatomic landmarks relating to the pulp chamber of the tooth. Great variance in overall molar tooth size, morphology, and position in the arch may have led to the assumption that the dimensions of the pulp chamber would also show such great variability that these measurements would be clinically useless.

In present study, variance is measured as a percentage of the mean (i.e. coefficient of variation [CV]_SD/mean). When comparing both maxillary and mandibular teeth, the range of CV values was $10 \%$ to $44.6 \%$. For mandibular first molars, the greatest $\mathrm{CV}$ values were observed for the pulp chamber height (measurement $F$ in Table 1). This inter-tooth variation in pulp chamber height is probably the result of the biologic process of secondary dentin apposition.

Several studies suggest that secondary dentin apposition occurs primarily on the pulp chamber floor instead of the ceiling. A study conducted by Philippas ${ }^{8)}$ evaluated ancient and contemporary populations and observed no significant changes in the dentin thickness at the roof of the pulp chamber with increasing age, whereas the dentin at the floor of the pulp chamber showed a definite increase in thickness with increasing age .

Another study conducted by Shaw and Jones ${ }^{9)}$ reported a highly significant reduction in the height of the pulp chamber of the mandibular first permanent molar (15\%) that was caused mainly by an increase in thickness of the pulpal floor. Tidmarsh ${ }^{10)}$ stated that "The growth of dentin upon the floors of pulp chambers, apparently without cause, is of some significance to the endodontist who must gain entrance to the root canals."

As this is a radiographic study, it is possible that variation in radiographic measurement versus anatomic measurement could be responsible for variation between groups. However, Scotti et al. ${ }^{11)}$ demonstrated that the residual error between the anatomic and radiographic measurement was $4.46 \%$, which, when extrapolated by Deutsch and Musikant $^{12)}$ is equivalent to an average difference of $0.12 \mathrm{~mm}$, this is very minimum and can not affects to this study results.

In the present study, mean distance measured between floor to closest point to the furcation (Measurement A) was 2.32 $0.47 \mathrm{~mm}$ which correlated with the finding obtained by a study by Khojastepour ${ }^{13)}(2.89 \pm 0.61)$, and Deutsch and 
Musikant ${ }^{12)}(2.96 \pm 0.78)$. While a study conducted by Velmurugan ${ }^{14)}$ reported that mean distance between floors to closest point to the furcation was $3.0 \pm 0.63$ which was quite high as compared to the present study.

In the present study mean distances from ceiling of pulp chamber to furcation (Measurement B) was $3.80 \pm 0.77 \mathrm{~mm}$. Study conducted by Deutsch and Musikant ${ }^{12)}(4.55 \pm 0.91)$ and Leila Khojastepour et al. ${ }^{13)}(5.22 \pm 0.85)$ also showed the similar finding. While study conducted by Velmurugan et al. ${ }^{14)}$ mention that the mean distances from ceiling of pulp chamber to furcation was $5.34 \pm 0.90 \mathrm{~mm}$.

The distance from buccal cusp to furcation (Measurement C) was $9.08 \pm 1.03 \mathrm{~mm}$. These finding are similar to finding obtained by most of the study. In the present study distance from buccal cusp to floor of pulp chamber (Measurement D) was $6.83 \pm 0.96 \mathrm{~mm}$ which is similar to the finding in a study by Deutsch \& Musikant ${ }^{12)}$ (7.95 \pm 0.79$)$. Distance from the buccal cusp to pulp chamber ceiling (Measurement E) was $5.37 \pm 0.79 \mathrm{~mm}$. These finding are similar to the findings obtained by Shaw and Jones ${ }^{9)}$, Deutsch and Musikant $^{12)}$.

While the height of pulp chamber (Measurement E) in the present study measured as $1.48 \pm 0.66 \mathrm{~mm}$ correlates with the finding obtained by Deutsch \& Musikant ${ }^{12)}(1.57 \pm 0.68)$ but the study by Khojastepour ${ }^{13)}$ showed higher value in the height of pulp chamber $(2.32 \pm 0.56 \mathrm{~mm})$.

The results of this study agree with the measurements reported by Sterrett et al. ${ }^{6}$, that the average distance from the pulp to the root surface (furcation) was $2.83 \pm 0.49 \mathrm{~mm}$ for mandibular first molars and $2.88 \pm 0.44 \mathrm{~mm}$ for mandibular second molars. Majzoub and $\mathrm{Kon}^{7)}$ measured maxillary molars and found that the distance from the pulp chamber floor to the most coronal aspect of the area of root separation (furcation) on the distal aspect was equal to or less than $3 \mathrm{~mm}$ in $86 \%$ of the teeth measured.

The reported measurements in present study and their similarity to measurements from other studies gives a general guideline for a more quantitative approach to endodontic molar access. In general, the distance from the cusp tip to pulp chamber ceiling height is approximately $6.0 \mathrm{~mm}$, the distance from the pulpal floor to the furcation is approximately $3.0 \mathrm{~mm}$, and the average height of a pulp chamber is 1.5 to $2.0 \mathrm{~mm}$. In addition, the pulp chamber ceiling was found at the level of the cementoenamel junction in $97 \%$ to $98 \%$ of the mandibular first molars.

This knowledge of pulp chamber morphology should be integrated with an examination of preoperative radiographs, to assess other potential factors such as taurodontism ${ }^{15}$.

\section{Conclusion}

The success of root canal treatment depends on triad of proper access opening through biomechanical preparation and three dimension obturation to produce air tight water tight seal. Hence, all step of root canal treatment must be done meticulously. Intraoral periapical radiograph can be used as the best tool to achieve successful results. Proper interpretation of intraoral periapical radiograph can avoid iatrogenic accidents such as perforations, which ultimately help in successful outcome of treatment.

\section{References}

1. Bhaskar SN: Orban's oral histology and embryology, 11th ed. St. Louis: CV Mosby; 1991: p.178.

2. Aguirre R, elDeeb ME, elDeeb ME. Evaluation of the repair of mechanical furcation perforations using amalgam, gutta-percha, or indium foil. J Endod. 1986; 12: 249-56.

3. Christie WH, Thompson GK. The importance of endodontic access in locating maxillary and mandibular molar canals. J Can Dent Assoc. 1994; 60: 527-32, 535-6.

4. Alhadainy HA. Root perforations, a review of literature. Oral Surg, Oral Med, Oral Pathol. 1994; 78: 368-74.

5. Goon WW, Lundergan WP. Redemption of a perforated furcation with a multidisciplinary treatment approach. J Endod. 1995; 21: 576-9.

6. Sterrett JD, Pelletier H, Russell CM. Tooth thickness at the furcation entrance of lower molars. J Clin Periodontol. 1996; 23: 621-7.

7. Majzoub Z, Kon S. Tooth morphology following root resection procedures in maxillary first molars. J Periodontol. 1992; 63: 290-6.

8. Philippas GC. Influence of occlusal wear and age on formation of dentin and size of pulp chamber. J Dent Res. 1961; 40: 1186-98.

9. Shaw L, Jones AD. Morphological considerations of the dental pulp chamber from radiographs of molar and premolar teeth. J Dent. 1984; 12: $139-45$.

10. Tidmarsh BG. Micromorphology of pulp chambers in human molar teeth. Int Endod J. 1980; 13: 69-75.

11. Scotti R, Villa L, Carossa S. Clinical applicability of the radiographic method for determining the thickness of calcified crown tissues. J Prosthet Dent. 1991; 65: 65-7. 


\section{References}

12. Deutsch AS,Musikant BL. Morphological measurement of anatomic landmarks in human maxillary and mandibular molar pulp chambers. $\mathrm{J}$ Endod. 2004; 30: 388-90.

13. Khojastepour L, Rahimizadeh N, Khayat A. Morphologic measurement of anatomic landmarks in pulp chambers of human first molar: a study of bitewing radiographs. Iranian Endod J. 2008; 1: 147-51.
14. Velmurugan N, Venkateshbabu N, Abarajithan M, Kandaswamy D. Evaluation of the pulp chamber size of human maxillary first molars: an institution based in vitro study. Indian J Dent Res. 2008; 19: 92-4.

15. Tsesis I, Shifman A, Kaufman AY. Taurodontism, an endodontic challenge: report of a case. J Endod. 2003; 29: 353-5. 